\title{
Lightning surge on the DC and AC side of solar PV system
}

\begin{abstract}
The solar PV has become an alternative solution in Malaysia to generate electricity. Unfortunately the solar PV installed in Malaysia is attracted to the lightning strike. There were two points that have high possibilities of being strike by the lightning; on the DC side between the solar PV and inverter and on the AC side, between the inverter and the substation. Therefore, this study was performed to analyse the effects of lightning strike at these two different points at the solar PV farm without any lightning protection system (LPS).
\end{abstract}

Keyword: Solar PV; Lightning current; Striking point; Transient voltage; Inverter; DC; AC 